\title{
Martha E. Sosa Torres and Peter M. H. Kroneck (Guest Editors), Astrid Sigel, Eva Freisinger and Roland K. O. Sigel (Series Editors): Transition metals and sulfur: a strong relationship for life, Vol. 20 of metal ions in life sciences
}

\author{
Walter de Gruyter, Berlin, Germany, 2020, 455 + xlv pp, ISSN: 1559-0836; 1868-0402 \\ (electronic). ISBN: 978-3-11-058889-7; 978-3-11-058975-7 (electronic PDF); 978-3-11-058894- \\ 1 (electronic EPUB). DOI: 10.1515/9783110588897. Walter de Gruyter, GmbH, Berlin, Germany, \\ 2020
}

\section{Nicolai Burzlaff ${ }^{1}$ (B)}

Received: 16 October 2020 / Accepted: 27 October 2020 / Published online: 27 November 2020 (c) The Author(s) 2020

Biological transition metal-sulfur sites are known for almost all so-called essential transition metal ions. Metalloenzymes based on such metal-sulfur sites catalyze some red-hot chemical reactions and a better understanding of the related processes might therefore solve existential problems of mankind in future. Enzymes with metal-sulfur sites promising such an impact are for example nitrogenases with P-cluster and a [Mo-7Fe-9S-C]-homocitrate catalytic site or carbon monoxide dehydrogenases and hydrogenases with $\mathrm{NiFeS}$ sites, just to name a few examples. Therefore, the 20th volume of the 'Metal Ions in Life Sciences' series edited by 'the Sigels' (Astrid Sigel, Eva Freisinger, Roland K.O. Sigel) is dedicated to sulfur and metal-sulfur sites and covers many aspects and a tremendous amount of information one could hardly extract and compile oneself in 12 chapters. Thus, this 20th volume certainly fills a gap in the metal ions in life science field and answers several questions a lecturer could be confronted with, in bioinorganic courses.

Chapter 1 by the volume editors Martha E. Sosa Torres and Peter M. H. Kroneck themselves resembles more or less an appetizer for this volume. In this introductory chapter, the sulfur-based focus of the volume is defined which clearly lies in the blue copper proteins, the $\mathrm{Cu}_{\mathrm{A}}$ copper-sulfide centre

Nicolai Burzlaff

nicolai.burzlaff@fau.de

Department of Chemistry and Pharmacy, FriedrichAlexander-University of Erlangen-Nürnberg, Egerlandstraße 1, 91058 Erlangen, Germany of cytochrome c oxidase (COX) or nitrous oxide reductase $\mathrm{N}_{2} \mathrm{OR}$, the tetranuclear copper-sulfide center $\mathrm{Cu}_{\mathrm{Z}}$ of $\mathrm{N}_{2} \mathrm{OR}$, the cytochrome $\mathrm{P}_{450}$ enzyme superfamily, iron sulfur clusters such as the siroheme-[4Fe-4S] centre, molybdenum and tungsten enzyme sites bearing the pterin dithiolene cofactor as well as the enzyme sites already mentioned above. Moreover, evolutionary connections and possible future directions are highlighted here.

Chapter 2 was also authored by Martha E. Sosa Torres and Peter M. H. Kroneck in cooperation with Alfonso R. Morales and Alejandro S. Peralta. It starts with a small essay about the various faces sulfur can exhibit in mineralogy and bio-mineralization as well as in organic and inorganic chemistry. This leads to microbial sulfur metabolism and aerobic oxidation as well as anaerobic reduction of sulfur for energy conservation purposes. This is exemplary discussed by means of a sulfur oxygenase and a polysulfide reductase. Microbial sulfate activation via adenosine 5'-phosphosulfate (APS) and sulfite reductases fit in this context, too. Then, cofactors and coenzymes with sulfur are reviewed such as biotin and thiamine. The chapter is then completed with the S-adenosyl-L-methionine (SAM) and its role in the radical SAM pathway.

Chapter 3 by Trinidad Arcos-López, Nils Schuth and Liliana Quintanar focuses on the type 1 blue copper (T1 Cu) sites in biology, especially the electron transfer (ET) properties of these sites and their unique spectroscopic features. As summarized nicely by the authors, these are controlled by entatic state geometry and a highly covalent $\mathrm{Cu}-\mathrm{S}$ bond 
with strong overlap between the metal $3 \mathrm{~d}_{\mathrm{x}^{2}-\mathrm{y}^{2}}$ orbital and the $S$ (Cys) 3p orbital. These features of the classic blue copper sites, such as azurin and plastocyanin, are then compared to those of so-called perturbed blue copper sites, including rusticyanin (Rc), pseudoazurin (Paz), cucumber basic protein (CBP) and nitrite reductases (NiR). By this comparison and a discussion of various studies with mutated $\mathrm{T} 1 \mathrm{Cu}$ sites, the authors succeeded to carve out how the axial ligand modulates the geometric and electronic structure of a blue copper protein. Besides discussing inner versus outer coordination sphere effects, this chapter finally focuses on biosynthetic blue copper centers, e.g. by converting other metalloenzymes such as zinc enzymes into BCP models. The chapter ends with a quite detailed description of electron transfer pathways and the tuning of type 1 copper reactivity for the specific biological function achieved by these various effects.

Chapter 4 by Marcos N. Morgada, Daniel H. Murgida, and Alejandro J. Vila deals with a related topic, the purple mixed-valent copper $\mathrm{A}\left(\mathrm{Cu}_{\mathrm{A}}\right)$ sites suitable for dioxygen and nitrous oxide reduction. Here a summary of native $\mathrm{Cu}_{\mathrm{A}}$ sites of various cytochrome oxidases (COXs) and nitrous oxide reductases $\left(\mathrm{N}_{2} \mathrm{ORs}\right)$ is a starting point for this chapter. Then mainly spectroscopic models derived by engineering the $\mathrm{Cu}_{\mathrm{A}}$-binding site into cupredoxin scafolds as well as synthetic model complexes are referred. This is followed by comments regarding the biogenesis of the $\mathrm{Cu}_{\mathrm{A}}$ sites in COXs and $\mathrm{N}_{2} \mathrm{ORs}$. Consequentially, based on a theoretical description, the spectroscopic properties of the native sites are compared to those of the models. This allows a discussion about first and second sphere effects that modulate the redox properties and potentials and subsequently affect the electron transfer dynamics and pathways. It is worthwhile mentioning that this part of the chapter contains some really educative figures. Finally, the authors lecture on the flexibility of the cupredoxin and the $\mathrm{Cu}_{\mathrm{A}}$ metal-binding regions and a frictionally controlled ET mechanism, caused by the high viscosities of the cell interior, resulting in significantly lower ET rates compared to those expected by the Marcus theory.

Chapter 5 carries on the topic but with a focus on the tetranuclear copper-sulfide center $\mathrm{Cu}_{\mathrm{Z}}(4 \mathrm{Cu} 1 \mathrm{~s})$, the active site of $\mathrm{N}_{2} \mathrm{ORs}$. The authors, Sofia R. Pauleta, Maria S. P. Carepo, and Isabel Moura, start with a general compendium covering the properties, the reactions with and the sources of nitrous oxide $\mathrm{N}_{2} \mathrm{O}$ as well as the denitrification pathway. This is followed by a review on the differences between Clade I and Clade II $\mathrm{N}_{2} \mathrm{ORs}$, the protein structures and the importance of a functional dimer formation for the catalytic activity. The authors then zoom in and report on the structure of the catalytic $\mathrm{Cu}_{\mathrm{Z}}$ enter, the redox and spectroscopic properties and the hypothesis of an unready state in case of Clade I $\mathrm{N}_{2} \mathrm{ORs}$ that might require an activation. Moreover, the potential $\mathrm{N}_{2} \mathrm{O}$ binding site in the $\mathrm{Cu}_{\mathrm{Z}}$ center is discussed, based on crystallographic studies with $\mathrm{N}_{2} \mathrm{O}$ and other compounds. This leads to the proposed catalytic cycle of $\mathrm{N}_{2} \mathrm{O}$ reduction by $\mathrm{N}_{2} \mathrm{OR}$ with the $\mathrm{Cu}_{Z}(4 \mathrm{Cu} 1 \mathrm{~S})$ site.

Chapter 6 authored by F. Miguel Castro Martinez, R. Daniel Páez López, Pedro D. Sarmiento Pavia, Martha E. Sosa Torres, and Peter M. H. Kroneck reviews the cytochrome $\mathrm{P}_{450}$ superfamily (CYPs, P450s): Nature's most versatile catalyst, as correctly stated by the authors. Taking into account the vast literature in this field, it was a very wise move of the authors to recommend several reprehensive review articles covering this field at the beginning, since this kept the introduction compact and short. Afterwards, the versatility, vast substrate range and diverse types of molecular transformations of CYPs are highlighted. Also, the variety of redox partners acting as essential electron sources for the catalytic cycle are disclosed. The next topic covered by this chapter is the engineering and directed evolution of enzymes, for which several reports based on P450s can serve as perfect textbook examples. Usually, such bioengineering studies are performed to achieve a defined regio- and/or stereo-selectivity, as pointed out by some prominent examples. According to the authors, some of the engineered CYPs are applied to the production of fine chemicals, sometimes even on industrial scale. Other modifications have the purpose to investigate the functional and structural properties in more detail. Since the parameters for such engineering studies are defined by the protein structure and its flexibility, the active site cavity and the axial cysteine ligand, these topics are discussed next. Afterwards, the focus of this chapter is drawn to the multiple redox partners, transferring electrons to the $\mathrm{P} 450$ heme center, such as the cytochrome $\mathrm{P} 450$ reductases which contain FAD and FMN redox cofactors or oxido-reductases and oxygenase reductases with $\mathrm{Fe}-\mathrm{S}$ cluster. Finally, the $\mathrm{C}-\mathrm{H}$ bond cleavage after dioxygen activation and all details of the $\mathrm{P} 450$ catalytic cycle are explained including spectroscopic and X-ray experiments to monitor P450 at work.

Basic iron-sulfur centers are reviewed by Claudia Andreini and Simone Ciofi-Baffoni in chapter 7. The introduction starts with the basic types of Fe-S clusters in proteins, namely $\mathrm{Fe}(\mathrm{S}-\mathrm{Cys})_{4}$, the $[2 \mathrm{Fe}-2 \mathrm{~S}]$, the $[3 \mathrm{Fe}-4 \mathrm{~S}]$ and the most common [4Fe-4S] cluster. Moreover, it covers the rapidly increasing number of available $\mathrm{Fe}-\mathrm{S}$ structures in the PDB database and some prominent examples such as the high potential iron proteins (HiPIP) are picked out, too. Subsequently, a more biochemical part talks about pathways of $\mathrm{Fe}-\mathrm{S}$ proteins biosynthetic systems, namely the iron-sulfur cluster (ISC) machinery and the sulfur mobilization (SUF) machinery. Starting with the Fe-S cluster biosynthesis and its transfer to client proteins in bacteria, this is later extended to corresponding processes in eukaryotes. For the latter also a cytosolic iron-sulfur protein assembly (CIA) machinery is explained. But most of this chapter deals with the occurrence 
and function of iron-sulfur proteins in organisms by listing anaerobic as well as aerobic organisms with a high content of Fe-S proteins in three very elaborate tables. Thus, this chapter, in contrast to all other ones, is less educative and more a compilation. The chapter ends with a short but very interesting passage about diseases associated with nuclear $\mathrm{Fe}-\mathrm{S}$ cluster-containing proteins.

In chapter 8, the 'Einsle' crew, namely Ivana Djurdjevic, Christian Trncik, Michael Rohde, Jakob Gies, Katharina Grunau, Florian Schneider, Susana L. A. Andrade, and Oliver Einsle, reports on the cofactors of nitrogenases and its role in the biological nitrogen fixation. Accordingly, the first part of this chapter is mainly structure-based, after briefly depicting the interplay between the homodimeric reductase (Fe protein) with the homodimeric MoFe protein. The following structure-based discussions focus on the architecture of dinitrogenases, the $[4 \mathrm{Fe}-4 \mathrm{~S}]$ cluster of the $\mathrm{Fe}$ protein as well as the $\mathrm{P}$ cluster (an $[8 \mathrm{Fe}-7 \mathrm{~S}]$ moiety) and the famous FeMo Cofactor, both embedded in the MoFe protein. The authors dedicated a major part of the chapter to this FeMo cofactor (an [Mo-7Fe-9S-C]-homocitrate moiety) in which the seminal discovery and identification of the central atom $\mathrm{X}$ - a carbide - is narrated elaborately. This story represents a wonderful example for the fruitful cooperation between crystallographers and experts in spectroscopy, biochemistry, biology and theoretical chemistry. Although a more detailed and antithetical discussion of the possible nitrogenase catalysis mechanism would have been helpful, the constraint to a short description of the Lowe-Thorneley mechanism is most likely owed to the page restrictions of this volume. The chapter ends with a part describing alternative nitrogenase systems, especially the vanadium nitrogenase with the $\mathrm{FeV}$ cofactor. Again, the high number of very educative figures in this chapter is worthwhile mentioning.

Chapter 9 by Khadanand KC and Martin L. Kirk then describes the pterin dithiolene cofactors, coordinated either to molybdenum or tungsten, and the reactions they catalyze, such as oxygen atom transfer (OAT) or hydroxylation. Since the molybdopterin cofactor (Moco) is essential for the more important molybdenum enzyme family, the authors refer in the beginning the Moco biosynthesis pathway starting from guanosine triphosphate (GTP). Moreover, questions regarding the Moco maturation and the effect of the sulfur incorporation as sulfido $\mathrm{Mo}=\mathrm{S}$ are discussed. In the following examples such as sulfite oxidase, the dimethylsulfoxide reductase, and the xanthine oxidase are reviewed, hereby especially considering the spectroscopic features, the electronic structures and the mechanistic aspects of these enzymes.

In Chapter 10, Isabel Askenasy and M. Elizabeth Stroupe pick out the siroheme-[4Fe-4S] coupled center as central topic. This chapter leads the reader back to the iron sulfur clusters discussed in chapter 7 . The chapter starts with the biogeochemical sulfur cycle and sulfur in biology and biochemistry. This is accompanied by a sequence and structure-based discussion regarding sulfite reductases that are siroheme-dependent (SiRHP). Siroheme is an iron-containing isobacteriochlorin with eight carboxylic groups and unique spectral properties. In the following SiRHP is compared to related enzymes based on X-ray crystallographic analyses and spectroscopic investigations. The chapter closes with the siroheme biogenesis.

Nickel, Iron and sulfur sites are reviewed by Yulia Ilina, Berta M. Martins, Jae-Hun Jeoung, and Holger Dobbek in Chapter 11. The chapter starts with the overall structure and the active site structure of the [NiFe] hydrogenases. The authors then report on the dihydrogen oxidation, which is a reversible process. Also, the catalytic cycle, the inhibition, and the photo-induction mechanisms for [NiFe] hydrogenases are discussed. Other enzymes covered in this chapter are the $[\mathrm{Ni4} F \mathrm{Fe}-4 \mathrm{~S}]$ carbon monoxide dehydrogenases that catalyze the $\mathrm{CO}$ oxidation. Here the reports regarding the structure and the ligand binding site of the $[\mathrm{Ni} 4 \mathrm{Fe}-4 \mathrm{~S}]$ cluster are reviewed. The reversible $\mathrm{CO}_{2}$ activation by nickel and iron and the mechanism of the $\mathrm{CO}$ oxidation at the cluster are discussed, too. Given the importance and the potential impact of both processes, this chapter truly deserves to be part of this volume. Also, the acetyl-coenzyme A synthases with a [2Ni4Fe-4S] site are included in this chapter.

Finally, in Chapter 12 the authors Gaetano Malgieri, Luigi Russo, Gianluca D’Abrosca, Ilaria Baglivo, Paolo V. Pedone, Roberto Fattorusso, and Carla Isernia pick up the well-known zinc finger (ZF) domains as a topic. The $\mathrm{ZF}$ domain is, according to the authors, a solid scaffold that originally had a DNA-binding role. Therefore, this topic differs from all the others of this volume that are somehow connected to redox chemistry. Apart from DNA binding, ZFs can have various specialized functions such as zinc sensing, protein folding, chromatin re-modeling, gene transcription, protein translation, and several others. This chapter resembles a nice summary of these topics.

In summary, the 20th volume of the 'Metals Ions in Life Sciences' series gives a comprehensive overview on the most important metalloenzymes and metalloproteins based on metal-sulfur sites. Due to specifications in time and space the volume editors as well as the authors had to restrict themselves tremendously and did this extremely well. In my opinion, every bioinorganic chemist interested in metalloenzymes and proteins will find some of her/his 'best buddies' in this selection. Moreover, most of the chapters and many of the figures collected from literature are very educative and will be very useful to decorate lectures for advanced students. Thus, the audience for this volume will certainly be very broad and there will be lots of unknown and essential facts every reader can extract 
from this compendium. I strongly recommend to acquire this volume for your private library. It certainly will have a special place on my bookshelf so that I can access it easily at any time.

Funding Open Access funding enabled and organized by Projekt DEAL.

Open Access This article is licensed under a Creative Commons Attribution 4.0 International License, which permits use, sharing, adaptation, distribution and reproduction in any medium or format, as long as you give appropriate credit to the original author(s) and the source, provide a link to the Creative Commons licence, and indicate if changes were made. The images or other third party material in this article are included in the article's Creative Commons licence, unless indicated otherwise in a credit line to the material. If material is not included in the article's Creative Commons licence and your intended use is not permitted by statutory regulation or exceeds the permitted use, you will need to obtain permission directly from the copyright holder. To view a copy of this licence, visit http://creativecommons.org/licenses/by/4.0/.

Publisher's Note Springer Nature remains neutral with regard to jurisdictional claims in published maps and institutional affiliations. 